\title{
Prevalência da síndrome de Burnout em anestesiologistas de Instituição de Ensino Superior em Medicina
}

\author{
Prevalence of Burnout syndrome in anesthetists from a Medical Education Institution
}

Fernando César Serralheiro', Alfésio Luiz Ferreira Braga², Maria Lúcia Bueno Garcia³, Thiago Grigio', Lourdes Conceição Martins ${ }^{1,2}$

\begin{abstract}
Resumo
Introdução: A moderna estrutura social vigente que, historicamente, privilegia as leis de mercado, atualmente se vê refletida, também, no âmbito profissional, uma vez que o indivíduo é avaliado a partir de parâmetros de produtividade e eficiência empresarial. A intensificação profissional cotidiana sobrecarregada acarreta importante redução do tempo disponível para si mesmo, facilitando amplo prejuízo em sua vida particular, favorecendo o aparecimento da síndrome de Burnout. Objetivo: Verificar a prevalência da síndrome de Burnout em anestesiologistas ligados à Faculdade de Medicina do ABC e sua relação com atividade física. Casuística e Métodos: Trata-se de um estudo transversal realizado por meio de dois questionários autoaplicados. Um abrangendo informações biodemográficas e o Maslach Burnout Inventory (MBI). Foi realizada a análise descritiva e o teste $\mathrm{U}$ de Mann-Whitney. Resultados: Observou-se que a maioria de anestesiologistas era do sexo masculino, casados e com filhos. $45,8 \%$ praticavam atividade física. Houve diferença apenas para o domínio despersonalização, no qual quem não praticava atividade física apresentou grau de despersonalização maior que os que têm essa prática rotineiramente (teste $U$ de Mann-Whitney, $p<0,05$ ). A maior parte dos anestesistas era de plantonistas e diaristas ao mesmo tempo e a maioria não conhecia o que é síndrome de Burnout. Conclusões: Embora a prevalência da síndrome nesse grupo de anestesistas tenha se mostrado baixa, ela e suas repercussões apontam para a urgente necessidade de intervenções institucionais com capacitação e apoio para que o profissional possa lidar com os aspectos objetivos e subjetivos da atividade assistencial específica da profissão.
\end{abstract}

Palavras-chave: análise transversal; esgotamento profissional; médicos.

\section{Abstract}

Introduction: Modern social structure prevailing today, favouring the laws of the market as throughout history, today also sees reflected under professional, being the professional evaluated from parameters of productivity and business efficiency. Objective: Verify the prevalence of Burnout among anesthesiologist connected the Faculty of Medicine of $A B C$ and its relationship with physical activity. Casuistry and Methods: Cross-sectional study conducted by applying two questionnaires. One about general informations and the other about Burnout syndrome (Maslach Burnout Inventory $(\mathrm{MBI}))$. The descriptive analysis was conducted and Mann-Whitney U test. Results: Most anesthesiologist laborers, almost most don't know what is Burnout. There is a prevalence of anesthetists male, married, with children. Almost $50 \%$ of anesthetists practice physical activity. There is a significant difference just for domain disidentification, where those who do not practice physical activity expose a degree of disidentification greater than those who practice (test of Mann-Whitney $U, p<0.05$ ). Conclusions: Burnout Syndrome and its repercussions point to the need for institutional interventions with training and support so that the trader can handle the subjective and objective aspects of activity specific assistential some professions.

Keywords: period analysis; burnout, professional; physicians.

Recebido em: 18/01/2011

Revisado em: 28/04/2011

Aprovado em: 21/10/2011

Trabalho realizado na Faculdade de Medicina do ABC (FMABC) - Santo André (SP), Brasil.

Programa de Pós-graduação em Ciências da Saúde da Faculdade de Medicina do ABC (FMABC) - Santo André (SP), Brasil

2 Programa de Pós-graduação em Saúde Coletiva da Universidade Católica de Santos (UNISANTOS) - Santos (SP), Brasil.

Programa de Pós-graduação em Ciências da Saúde da Faculdade de Medicina da Universidade de São Paulo (FMUSP) - São Paulo (SP), Brasil.

Endereço para correspondência: Lourdes Conceição Martins - Rua Abraham Bloemaert, 126 - Jardim das Vertentes - CEP: $05541-320$ -

São Paulo (SP), Brasil-E-mail: lourdesc@usp.br.

Fonte de Financiamento: nenhuma.

Conflito de interesse: nada a declarar. 


\section{Introdução}

A moderna estrutura social vigente que, historicamente, privilegia as leis do mercado, atualmente se vê refletida, também, no âmbito profissional, uma vez que o indivíduo é avaliado a partir de parâmetros de produtividade e eficiência empresarial. Nesse contexto, os médicos, como trabalhadores, passam a preocupar-se não só com suas funções clínicas, mas também com questões baseadas no paradigma da civilização industrial ${ }^{1}$.

Claramente, as atribuições impostas ao médico são muitas e, frequentemente, alheias aos seus interesses e, muitas vezes, à sua carga horária. Além do exercício clínico ou cirúrgico, desenvolve trabalhos administrativos, planeja ações, entre outras tarefas. Além disso, tem de reciclar-se, investigar, organizar, participar de reuniões, seminários, conselhos, processos de avaliação além do preenchimento de inúmeros relatórios periódicos e individuais ${ }^{1}$.

Entretanto, essa intensificação profissional cotidiana sobrecarregada acarreta importante redução do tempo disponível para si mesmo, facilitando amplo prejuízo em sua vida particular.

Criado na década de setenta, pela psicóloga Cristina Maslach, o conceito de Burnout, apesar de algumas críticas iniciais, foi rapidamente aceito e tem sido tema de inúmeros artigos científicos, livros e apresentações em congressos de psicologia, psiquiatria e educação médica, tornando-se assim um conceito inquestionável ${ }^{1}$.

Essa síndrome é considerada por Harrison como um tipo de estresse de caráter duradouro, vinculado às situações de trabalho, sendo resultante da constante e repetitiva pressão emocional associada ao intenso envolvimento interpessoal por longos períodos de tempo, gerando esgotamento emocional, desenvolvimento de atitudes e sentimentos negativos para com as pessoas, bem como com o próprio papel profissional no tocante ao envolvimento pessoal ${ }^{2}$.

Há autores que definem o Burnout como uma síndrome ou estado. As tendências nos últimos anos apontam o Burnout como um processo caracterizado por: antecedentes, síndrome propriamente dita e suas consequências ${ }^{3}$.

No entanto, mais que os sinais e sintomas, o que caracteriza essa síndrome são seus fatores desencadeantes, a ligação direta com a frustração na busca de resultados e também sua incapacidade para reverter tal quadro. Tudo isso, aliado à falta de valorização social e de reconhecimento do seu trabalho ${ }^{4-6}$.

O objetivo deste estudo foi verificar a prevalência de Burnout entre anestesiologistas ligados à Faculdade de Medicina do ABC e sua relação com atividade física.

\section{Casuística e métodos}

Trata-se de um estudo do tipo transversal, realizado por meio de questionário autoaplicado e autoexplicativo não identificado, onde o avaliador não tomava conhecimento das respostas, fato de conhecimento prévio por parte do entrevistado. A população estudada foi com- posta por médicos anestesiologistas ligados à Faculdade de Medicina do ABC, após concordarem em participar e assinarem o Termo de Consentimento Livre e Esclarecido.

O projeto foi aprovado pelo Comitê de Ética em Pesquisa da Faculdade de Medicina do ABC, sob protocolo no 137/2009.

Foi utilizada uma amostra não probabilística, por conveniência, em que todos os anestesiologistas ligados à FMABC foram convidados a participar (70 médicos anestesiologistas), porém, concordaram em participar 59 (84\%) destes, no período de julho de 2009 a julho de 2010, após assinarem o Termo de Consentimento Livre e Esclarecido. A não participação foi justificada pela falta de tempo e grande número de pacientes para atender.

Dois questionários foram usados. Um que abrange esferas diversas da vida do sujeito, como: identificação, sexo, situação conjugal, idade, filhos, formação profissional e características de trabalho (natureza do vínculo, tempo de formado). O segundo questionário foi o Maslach Burnout Inventory (MBI).

O questionário MBI é constituído por 22 itens, divididos em três domínios fundamentais: exaustão emocional, despersonalização e envolvimento ou realização profissional ${ }^{3}$.

As perguntas do questionário fornecem informações importantes sobre variáveis presentes na vida dos sujeitos, que podem ser componentes atuantes na facilitação ou não do esgotamento emocional, da despersonalização e da realização/envolvimento profissional, ou seja, da geração da síndrome em si. A escala de resposta é do tipo Likert de sete pontos (desde zero $=$ "nunca" até seis $=$ "todos os dias") ${ }^{3}$.

Primeiramente, foram calculados os valores para cada um dos domínios do MBI. A seguir cada domínio foi estratificado em baixo, médio e alto, pela divisão em percentil, em que baixo estava no percentil $25 \%$, médio entre o percentil $25 \%$ e $75 \%$ e alto acima do percentil $75 \%$.

Para se conhecer o perfil final de Burnout desse grupo de estudo foi definido a variável final como sendo:

- Burnout alto (aquele indivíduo que tem alto esgotamento emocional, alta despersonalização e baixa realização/envolvimento profissional);

- Burnout médio (aquele indivíduo que tem médio esgotamento emocional, média despersonalização e média realização/envolvimento profissional);

- Burnout baixo (os demais).

A atividade física (sim/não) foi considerada como grupo de estudo. Realizou-se, também, a análise descritiva de todas as variáveis do estudo. As variáveis qualitativas apresentadas em termos de seus valores absolutos e relativos. As variáveis quantitativas foram apresentadas em termos de seus valores de tendência central e de dispersão ${ }^{7}$.

Para as variáveis quantitativas, a homogeneidade das variâncias e a aderência à curva normal foram verificadas pelos testes de Levene e Kolmogorov-Smirnov, respectivamente ${ }^{7,8}$. Como as variáveis não apresentaram esses dois princípios satisfeitos, foi utilizado o teste $\mathrm{U}$ de 
Mann-Whitney para comparar esses grupos. O nível de significância foi de $5 \%$.

\section{Resultados}

O questionário foi respondido por 59 (80\%) dos médicos anestesiologistas da FMABC.

A idade média do grupo foi de 43,5 (dp=9,1) anos, e o tempo médio de exercício profissional foi de 17,5 ( $\mathrm{dp}=9,1)$ anos.

A maior parte dos anestesiologistas eram plantonistas e diaristas.

Entre os entrevistados $56(94,9 \%)$ eram apenas especialistas, $2(3,4 \%)$ eram mestres e $1(1,7 \%)$ doutor.

A renda variou entre 10 a 100 salários mínimos, sendo que a maioria $(23,7 \%)$ dos profissionais tinham renda de 20 salários mínimos.

Houve uma prevalência de anestesiologistas do sexo masculino, casados e com filhos e a maioria não conhecia o que é Burnout.

$45,8 \%$ dos anestesistas praticavam atividade física, sendo que, destes, $88,9 \%$ praticavam de duas a três vezes por semana. As atividades físicas mais praticadas foram corrida $(29,6 \%)$ e caminhada $(22,2 \%)$.

A Tabela 1 apresenta a análise descritiva para sexo, jornada de trabalho, estado civil, conhecimento a respeito de Burnout, filhos e atividade física.

$\mathrm{Na}$ Tabela 2 é apresentada a análise descritiva por domínio do questionário MBI.

Observou-se que para o domínio esgotamento emocional (EE) houve uma variação entre 8 e 54 pontos, para despersonalização (DP) houve variação de 0 a 21 pontos e para o envolvimento/realização profissional (EP) a variação foi de 6 a 46 pontos.

Quanto à análise estratificada dos domínios do Burnout, observouse que a maior parte dos profissionais estudados apresenta grau médio para ambos os domínios - esgotamento emocional, despersonalização e envolvimento/realização profissional, conforme a Tabela 3.

Considerando o escore final de Burnout, temos que 47 (79,7\%) anestesiologistas apresentaram nível de Burnout baixo, 10 (16,9\%) apresentaram nível médio e $2(3,4 \%)$ apresentaram nível sugestivo de Burnout alto, mostrando que o nível de Burnout na população estudada é, prevalentemente, baixo.

A Tabela 4 apresenta a análise descritiva por domínios do questionário MBI estratificada por atividade física. Observou-se diferença apenas para o domínio despersonalização, no qual quem não pratica atividade física apresenta grau de despersonalização maior do que quem pratica (teste $\mathrm{U}$ de Mann-Whitney, $\mathrm{p}<0,05$ ).

\section{Discussão}

Este estudo mostrou que a maioria dos anestesiologitas desconhecem a síndrome de Burnout e também que esta é uma população crítica de prevalência dessa síndrome. A atividade física permitiu melhor resposta adaptativa do indivíduo a essa agressão, induzindo menor grau de despersonalização desses indivíduos. Esses dados identificam essa população como alvo dessa afecção e permitem sugerir que medidas preventivas são necessárias para amenizar os sintomas prejudiciais à qualidade de vida desses iundivíduos.

Burnout acometeu 100\% da população estudada. Após estratificação, constatou-se riscos de Burnout alto e médio, atingindo a maioria da população $(79,4 \%)$, quando analisados os três domínios conjuntamente.

Quando realizada a estratificação dos domínios do questionário MBI por atividade física, observou-se que os praticantes apresentaram nível de despersonalização menor que os não praticantes. Tal fato pode ser atribuído a uma maior convivência com outras pessoas.

A divisão por atividade física foi realizada por ser um fator importante na qualidade de vida, já que, sabidamente, combate o estresse, além de promover inúmeros outros benefícios à saúde?

Não se pode deixar de citar, também, que, embora a prevalência da síndrome se mostre pequena no grupo estudado, há de se enfatizar

Tabela 1: Análise descritiva de dados biodemográficos

\begin{tabular}{lrr}
\hline \multicolumn{1}{l}{$\mathrm{n}$} & $\%$ \\
\hline $\begin{array}{l}\text { Jornada de trabalho } \\
\text { Plantonista }\end{array}$ & 21 & 35,6 \\
$\quad$ Diarista & 5 & 8,5 \\
$\quad$ Plantonista e diarista & 33 & 55,9 \\
Conhecimento do Burnout & & \\
$\quad$ Sim & 14 & 23,7 \\
$\quad$ Não & 45 & 76,3 \\
\hline Sexo & & \\
$\quad$ Masculino & 31 & 52,5 \\
\hline Feminino & 28 & 47,5 \\
\hline Estado Civil & & \\
Casado & 32 & 54,2 \\
\hline Solteiro & 13 & 22,0 \\
\hline Divorciado & 14 & 23,7 \\
\hline Filhos & & 52,5 \\
\hline Sim & 31 & 47,5 \\
\hline Não & 28 & 45,8 \\
\hline Atividade Física & & 54,2 \\
\hline Sim & 27 & \\
\hline Não & 32 & \\
\hline
\end{tabular}

n: número

Tabela 2: Análise descritiva para os domínios do questionário Maslach Burnout Inventory

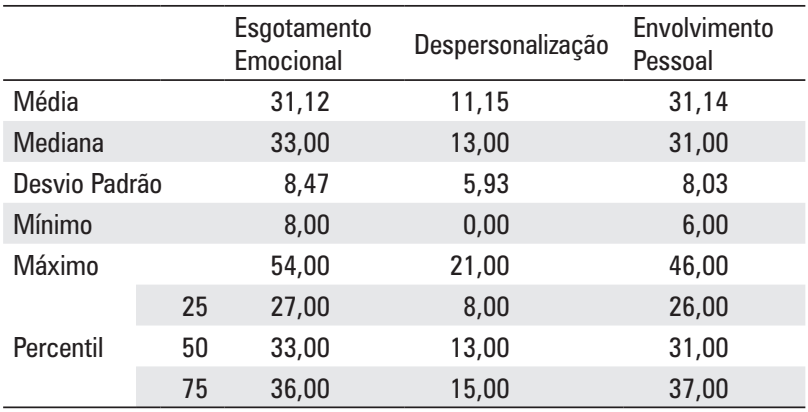


Tabela 3: Análise descritiva para o questionário Maslach Burnout Inventory por domínios

\begin{tabular}{lcc}
\hline & $\mathrm{n}$ & $\%$ \\
\hline $\begin{array}{l}\text { Esgotamento Emocional } \\
\text { Baixo }\end{array}$ & 11 & 18,64 \\
$\quad$ Médio & 30 & 50,85 \\
$\quad$ Alto & 18 & 30,51 \\
$\begin{array}{l}\text { Despersonalização } \\
\quad \text { Baixo }\end{array}$ & \\
$\quad$ Médio & 13 & 22,03 \\
$\quad$ Alto & 26 & 44,07 \\
Realização/Envolvimento Profissional & 20 & 33,90 \\
$\quad$ Baixo & & \\
$\quad$ Médio & 12 & 20,34 \\
$\quad$ Alto & 32 & 54,24 \\
\hline
\end{tabular}

n: número

a existência de indivíduos com alto grau no escore para síndrome de Burnout, gerando questionamento sobre o quanto essa síndrome pode afetar a importante segurança no exercício profissional diário, uma vez que a especialidade em foco exige enorme capacidade por parte do profissional.

Ainda, diante da baixa prevalência observada entre os 59 profissionais estudados, não foi possível excluir a possibilidade de que entre os $20 \%$ que se recusou a participar do estudo, a prevalência de Burnout alto fosse maior do que a encontrada entre os demais.

Cabe destacar que, embora tendo analisado uma amostra de profissionais, este estudo poderá ser extrapolado para profissionais da mesma especialidade que trabalham em situações semelhantes, como policiais, bombeiros, educadores, entre outros ${ }^{10}$.

Nos últimos anos, observou-se um aumento no interesse de pesquisadores sobre a síndrome de Burnout entre médicos. Um estudo realizado por Arigoni et al. ${ }^{9}$, na Suíça, observou-se prevalência de Burnout de 33 a $42 \%$ entre os médicos, sendo essa alta prevalência atribuída ao aumento da complexidade do sistema de saúde suíço, aumento que se mostra como tendência mundial' .

Entretanto, a prevalência de Burnout entre anestesiologistas no Brasil ainda não é conhecida. Lourenção et al. ${ }^{10}$ avaliaram o estresse e
Tabela 4: Análise dos domínios de Burnout por atividade física

\begin{tabular}{lccc}
\hline & \multicolumn{2}{c}{ Atividade Física } & Nível de \\
\cline { 2 - 4 } & \multicolumn{1}{c}{ Sim } & Não & Significância* \\
\hline Esgotamento Emocional & & & \\
Média (dp) & $29,7(10,8)$ & $32,3(5,7)$ & 0,39 \\
Mínimo - Máximo & $8-54$ & $11-44$ & \\
Despersonalização** & & & \\
Média (dp) & $9,4(6,1)$ & $12,7(5,4)$ & 0,03 \\
Mínimo - Máximo & $0-19$ & $0-21$ & \\
Realização/Envolvimento & & & \\
Profissional & & & \\
Média (dp) & $31,8(9,9)$ & $30,6(6,2)$ & 0,18 \\
\hline Mínimo - Máximo & $6-46$ & $19-46$ & \\
\hline
\end{tabular}

"Teste U de Mann-Whitney; " $p<0,05$; dp: desvio padrão

a qualidade de vida entre residentes de medicina na cidade de São José do Rio Preto e observaram que entre residentes há uma baixa qualidade de vida com aumento de estresse.

Contudo, ainda são raros os trabalhos que avaliam essa síndrome entre médicos. Nos últimos cinco anos, foram encontradas, nas bases de dados Pubmed, que é a maior base de dados de estudos na área das ciências biológicas, por volta de 40 artigos, destes, apenas 20 eram relacionados a médicos e 1 em anestesistas. Tal fato reforça a necessidade de novos estudos acerca dessa temática, vista a contribuição para o conhecimento da real prevalência do Burnout entre médicos brasileiros, além da criação de programas que busquem a melhoria das condições de saúde desses profissionais.

Em conclusão, este estudo mostrou uma baixa prevalência da síndrome de Burnout entre os anestesiologistas, porém, há casos da síndrome no grupo que apontam para a necessidade de reconhecimento amplo e generalizado dessa doença pela sociedade, possibilitando a implantação de intervenções institucionais nas diversas esferas, gerando capacitação e, sobretudo, apoio para que o profissional possa lidar com inúmeros aspectos objetivos e subjetivos inerentes à atividade assistencial, prevenindo-o e protegendo-o contra a instalação insidiosa e nefasta dessa síndrome e suas repercussões.

\section{Referências}

1. Carlotto MS, Palazzo LS. Síndrome de Burn out e fatores associados: um estudo epidemiológico com professores. Cad Saúde Pública. 2006; 22(5):1017-26.

2. Harrison BJ. Are you to Burnout? Fund Raising Manage. 1999;30:25-8.

3. Maslach C, Jackson SE. The measurement of experienced Burnout. J Occup Behav. 1981:2:99-113

4. Millan LR. A síndrome de Burnout: realidade ou ficção? Rev Assoc Med Bras. 2007:53(1):1-12

5. Nogueira-Martins MCF, Ramalho MA. Psicologia em estudo. Maringá: 2007;12(1):123-32.
6. Jimenez BM, Garrosa-Hernandez E, Gálvez M, et al. Psicologia em Estudo (Maringá). 2002;7(1):11-19.

7. Callegari-Jacques, SM. Bioestatística: princípios e aplicações. Porto Alegre: Artmed, 2003.

8. Siegel S. Estatística não paramétrica. São Paulo: McGraw-Hill do Brasil, 1981.

9. Arigoni F, Bovier P, Sappino AP. Trend in Burnout among Swiss doctors. Swiss Med Wkly. 2010;140:E1-E8.

10. Lourenção LG, Moscardini AC, Soler ZASG. Health and quality of life of medical residents. RAMB. 2010;56(1):81-90. 